KEK Preprint 2002-95

\title{
Connection Conditions and the Spectral Family under Singular Potentials
}

\author{
IzUmi Tsutsui* and TAMÁs FÜLÖP ${ }^{\dagger}$ \\ Institute of Particle and Nuclear Studies \\ High Energy Accelerator Research Organization (KEK) \\ Tsukuba 305-0801, Japan \\ and \\ TAKSU CHEON ${ }^{\ddagger}$ \\ Laboratory of Physics \\ Kochi University of Technology \\ Tosa Yamada, Kochi 782-8502, Japan
}

\begin{abstract}
To describe a quantum system whose potential is divergent at one point, one must provide proper connection conditions for the wave functions at the singularity. Generalizing the scheme used for point interactions in one dimension, we present a set of connection conditions which are well-defined even if the wave functions and/or their derivatives are divergent at the singularity. Our generalized scheme covers the entire $U(2)$ family of quantizations (self-adjoint Hamiltonians) admitted for the singular system. We use this scheme to examine the spectra of the Coulomb potential $V(x)=-e^{2} /|x|$ and the harmonic oscillator with square inverse potential $V(x)=\left(m \omega^{2} / 2\right) x^{2}+g / x^{2}$, and thereby provide a general perspective for these models which have previously been treated with restrictive connection conditions resulting in conflicting spectra. We further show that, for any parity invariant singular potentials $V(-x)=V(x)$, the spectrum is determined solely by the eigenvalues of the characteristic matrix $U \in U(2)$.
\end{abstract}

\footnotetext{
* email: izumi.tsutsui@kek.jp

$\dagger$ email: fulopt@poe.elte.hu

$\ddagger$ email: cheon@mech.kochi-tech.ac.jp, http://www.mech.kochi-tech.ac.jp/cheon/
} 


\section{Introduction}

Quantum singularity is a source of interesting physics and, at the same time, confusion. Even in its simplest form as a point interaction — now realized approximately as quantum dots (see, e.g., [1]) — it provides unexpectedly rich quantum phenomena such as duality and anholonomy [2]. When it arises as a divergent point of an infinite potential wall, it may admit quantum tunnelling through the infinite wall and, in some cases, can lead to an exotic quantum caustic [3]. However, it also poses the problem in its own treatment in quantum mechanics. In fact, if we look back the history of the one dimensional Coulomb potential, $V(x)=-e^{2} /|x|$, for instance, we find persistent disagreement over the possible spectrum for nearly a half century [4]. A similar confusion can be found for a system with the square inverse potential, $V(x)=g / x^{2}[5]$.

These confusing circumstances arise due to the ambiguity in choosing boundary (or connection) conditions at the singularity, which in mathematical terms corresponds to the choice of self-adjoint domains for the Hamiltonian operator,

$$
H=-\frac{\hbar^{2}}{2 m} \frac{\mathrm{d}^{2}}{\mathrm{~d} x^{2}}+V(x) .
$$

It has been known that a point interaction on a one dimensional line admits a $U(2)$ family of self-adjoint extensions for the Hamiltonian, and that these are characterized by distinct connection conditions [6]. For a point interaction occurring at $x=0$, the connection conditions can be given by

$$
(U-I) \Psi+i L_{0}(U+I) \Psi^{\prime}=0
$$

where $U \in U(2)$ is a matrix characterizing the self-adjoint extension, $I$ is the identity matrix, and $L_{0} \neq 0$ is a constant with dimension of length $[7,8,9]$. $\Psi$ and $\Psi^{\prime}$ are boundary vectors

$$
\Psi=\left(\begin{array}{c}
\psi(+0) \\
\psi(-0)
\end{array}\right), \quad \Psi^{\prime}=\left(\begin{array}{c}
\psi^{\prime}(+0) \\
-\psi^{\prime}(-0)
\end{array}\right)
$$

defined from the boundary values $\psi( \pm 0)=\lim _{x \rightarrow \pm 0} \psi(x)$ of the wave function $\psi$ and its derivative $\psi^{\prime} \equiv \frac{\mathrm{d} \psi}{\mathrm{d} x}$. The problem, however, is that this prescription of connection conditions may not be directly applicable to singular potentials $V(x)$, because then the boundary values $\psi( \pm 0)$ and/or $\psi^{\prime}( \pm 0)$ may diverge at the singularity and, accordingly, the vectors $\Psi$ and $\Psi^{\prime}$ in (3) become ill-defined.

One of the purposes of the present paper is to provide a scheme in which the connection conditions (2) become well-defined even for singular potentials, and thereby furnish a 
general framework for studying singular systems on a line including those mentioned above. Typically, at the singularity such systems allow for two independent square integrable solutions $x=0$ for the eigenvalue equation $H \psi=E \psi$ for any $E$, and (at most) only one square integrable solution at $x \rightarrow \pm \infty$. Systems of this type are said to be in the limit-circle case at $x=0$ and in the limit-point case at infinity [6]. At a limit-point singularity, no boundary condition is needed for ensuring the self-adjointness of the Hamiltonian [7], while at a limit-circle singularity, some boundary conditions are necessary in order to specify a self-adjoint Hamiltonian from the family of possible self-adjoint domains. This family is $U(2)$ for these systems, which follows, for example, from the fact that each of the negative and the positive half lines has one square integrable eigenmode for any nonreal eigenvalue $E[6,7]$. An essential step toward the generalization of the connection conditions for such systems consists of replacing the boundary values of the wave functions with corresponding Wronskians. This idea has been proposed [10] for systems on a half line with a singular endpoint for which a $U(1)$ family of boundary conditions is assigned. Here, we extend this to the full line, where now the family is given by $U(2)$, in such a way that the connection conditions (2) remain to be valid with modified boundary vectors. (For a different scheme of providing the domains of possible self-adjoint Hamiltonians, see [11].)

For illustration, we employ our scheme to analyze two models, one with the Coulomb potential and the other with the harmonic oscillator with square inverse potential $V(x)=$ $\left(m \omega^{2} / 2\right) x^{2}+g / x^{2}$. We shall see that the various different quantizations discussed previously for those models arise at different choices of the matrix $U$, and that the spectra are dependent on the choice of $U$; in fact, this dependence has caused the confusion concerning the spectrum in the literature. Interestingly, the spectrum depends on two parameters (the eigenvalues of the matrix $U$ ), not all the four of $U \in U(2)$, and this two-parameter dependence of the spectra is shared by any parity invariant potentials $V(-x)=V(x)$ with singularity of the kind just mentioned. More precisely, we find that for those systems the space of spectra is given by the Möbius strip $U(1) \times U(1) / \mathbb{Z}_{2}$.

The plan of this paper follows the line of arguments stated here, that is, we give the generalized connection conditions in Sect. 2 and thereby analyze the two models in Sect. 3. The two-parameter dependence of the spectra is then established in Sect.4, and finally Sect. 5 is devoted to summary and discussions. 


\section{Connection conditions}

To begin with, we provide the general connection conditions by extending the construction proposed in Ref. [10] (see also $[12,13,14]$ ) from the half line to the full line. Let the potential $V$ possess a singularity at $x=0$ on the one dimensional line $\mathbb{X} .{ }^{1}$ The potential is assumed to be in the limit-circle case at $x=0$ from both sides and in the limit-point case at $x \rightarrow \pm \infty$, and regular otherwise. We first consider the maximum domain $\mathcal{F} \subset L^{2}(\mathbb{X})$ on which the Hamiltonian $H$ can be defined as a differential operator,

$$
\begin{aligned}
\mathcal{F}=\left\{\psi \in L^{2}(\mathbb{X}) \mid \psi \text { and } \psi^{\prime}\right. \text { are absolutely continuous on } \\
\text { every finite subinterval of } \left.\mathbb{X} \backslash\{0\}, \quad H \psi \in L^{2}(\mathbb{X})\right\} .
\end{aligned}
$$

The Hamiltonian is not symmetric on $\mathcal{F}$, since, for $\phi, \psi \in \mathcal{F}$, we have

$$
\int_{\mathbb{X}} \mathrm{d} x\left[\phi^{*} H \psi-(H \phi)^{*} \psi\right]=\frac{\hbar^{2}}{2 m}\left(W\left[\phi^{*}, \psi\right]_{+0}-W\left[\phi^{*}, \psi\right]_{-0}\right),
$$

where $W\left[\phi^{*}, \psi\right]_{ \pm 0}$ are the limiting values for $x \rightarrow \pm 0$ of the Wronskian

$$
W\left[\phi^{*}, \psi\right](x)=\phi^{*}(x) \psi^{\prime}(x)-\phi^{* \prime}(x) \psi(x) .
$$

Here we have utilized the facts that $W\left[\phi^{*}, \psi\right]$ vanishes for $x \rightarrow \pm \infty$, since the infinites are limit-point [7], and that it is finite in the limits $x \rightarrow \pm 0$ even if the two functions $\phi(x)$, $\psi(x)$ are divergent. This latter can be shown as follows. For $\epsilon>0$, we introduce the space of functions

$$
\begin{aligned}
\mathcal{F}_{\epsilon}=\left\{\psi \in L^{2}(0, \epsilon) \mid \psi \text { and } \psi^{\prime}\right. \text { are absolutely } \\
\text { continuous on } \left.(0, \epsilon), H \psi \in L^{2}(0, \epsilon)\right\} .
\end{aligned}
$$

Note that $\mathcal{F}_{\epsilon}$ contains $\mathcal{F}$ as well as a wide range of other interesting functions as well, including all the eigenfunctions of the differential operator $H$, which are square integrable in any finite neighbourhood of the limit-circle singularity $x=0$ but not necessarily on the whole line $\mathbb{X}$. Now, for $\phi, \psi \in \mathcal{F}_{\epsilon}$ and $0<\delta<\epsilon$,

$$
\int_{\delta}^{\epsilon} \mathrm{d} x\left[\phi^{*} H \psi-(H \phi)^{*} \psi\right]=\frac{\hbar^{2}}{2 m}\left(W\left[\phi^{*}, \psi\right]_{\delta}-W\left[\phi^{*}, \psi\right]_{\epsilon}\right) .
$$

Both terms of the r.h.s. are finite. The l.h.s. is also finite, even if we let $\delta \rightarrow 0$. Consequently, $\lim _{\delta \rightarrow 0} W\left[\phi^{*}, \psi\right]_{\delta}=W\left[\phi^{*}, \psi\right]_{+0}$ is finite. As one can see, this property holds actually not only in $\mathcal{F}$ but even in $\mathcal{F}_{\epsilon}$. The finiteness of $W\left[\phi^{*}, \psi\right]_{-0}$ is proved similarly, with the aid of the analogously introduced $\mathcal{F}_{-\epsilon}$.

\footnotetext{
1 We use the symbol $\mathbb{X}$ to stress that it is dimensionful in contrast to the dimensionless real line $\mathbb{R}$.
} 
Since the r.h.s. of (5) is generally nonvanishing, any self-adjoint domain $\mathcal{D}$ for $H$ must be such a subset of $\mathcal{F}$ that the r.h.s. of (5) is zero for all functions within $\mathcal{D}$. Now we show how to characterize the possible self-adjoint domains via a connection condition at $x=0$ of the form (2), where the boundary vectors $\Psi, \Psi^{\prime}$ are appropriately generalized with the help of a basic set of energy eigenmodes. Let $\varphi^{(i)}$, for $i=1,2$, be two independent, real eigenmodes with eigenvalue $E$,

$$
H \varphi^{(i)}(x)=E \varphi^{(i)}(x), \quad W\left[\varphi^{(1)}, \varphi^{(2)}\right](x)=1,
$$

for $x \neq 0$. The actual value of $E$ is unimportant for our purposes. We note that these eigenmodes may not be square integrable on the whole line and hence may not belong to $\mathcal{F}$, but they necessarily belong to $\mathcal{F}_{\epsilon}$ and $\mathcal{F}_{-\epsilon}$. Consequently, the complex column vectors

$$
\Psi=\left(\begin{array}{l}
W\left[\psi, \varphi^{(1)}\right]_{+0} \\
W\left[\psi, \varphi^{(1)}\right]_{-0}
\end{array}\right), \quad \Psi^{\prime}=\left(\begin{array}{c}
W\left[\psi, \varphi^{(2)}\right]_{+0} \\
-W\left[\psi, \varphi^{(2)}\right]_{-0}
\end{array}\right)
$$

are well-defined for $\psi \in \mathcal{F}$ since they are constructed from finite quantities. Further, observing that we can rewrite the Wronskian (6) as

$$
\begin{aligned}
W\left[\phi^{*}, \psi\right] & =\left|\begin{array}{cc}
\phi^{*} & \phi^{*^{\prime}} \\
\psi & \psi^{\prime}
\end{array}\right|=\left|\begin{array}{cc}
\phi^{*} & \phi^{*^{\prime}} \\
\psi & \psi^{\prime}
\end{array}\right|\left|\begin{array}{cc}
\varphi^{(1)^{\prime}} & \varphi^{(2)^{\prime}} \\
-\varphi^{(1)} & -\varphi^{(2)}
\end{array}\right| \\
& =\left|\begin{array}{cc}
\phi^{*} \varphi^{(1)^{\prime}}-\phi^{*^{\prime}} \varphi^{(1)} & \phi^{*} \varphi^{(2)^{\prime}}-\phi^{*^{\prime}} \varphi^{(2)} \\
\psi \varphi^{(1)^{\prime}}-\psi^{\prime} \varphi^{(1)} & \psi \varphi^{(2)^{\prime}}-\psi^{\prime} \varphi^{(2)}
\end{array}\right| \\
& =W\left[\phi^{*}, \varphi^{(1)}\right] W\left[\psi, \varphi^{(2)}\right]-W\left[\phi^{*}, \varphi^{(2)}\right] W\left[\psi, \varphi^{(1)}\right],
\end{aligned}
$$

we can express the r.h.s. of (5) in terms of the boundary vectors for $\phi$ and $\psi$ simply as

$$
\frac{\hbar^{2}}{2 m}\left[\Phi^{\dagger} \Psi^{\prime}-\Phi^{\prime \dagger} \Psi\right]
$$

where $\Phi$ and $\Phi^{\prime}$ are introduced for $\phi$ analogously to (10) for $\psi$. If $\phi, \psi$ are in a selfadjoint domain $\mathcal{D}$ then (12) must vanish. In particular, for $\phi=\psi$, this condition reads $\Psi^{\dagger} \Psi^{\prime}=\Psi^{\prime \dagger} \Psi$, which, under the notations

$$
\Psi^{( \pm)}=\Psi \pm i L_{0} \Psi^{\prime}
$$

with an arbitrary nonzero constant $L_{0}$, is equivalent to the equality of the norms $\left\|\Psi^{(+)}\right\|=$ $\left\|\Psi^{(-)}\right\|$. This shows that $\Psi^{(+)}$and $\Psi^{(-)}$are in a relationship

$$
U \Psi^{(+)}=\Psi^{(-)}, \quad U \in U(2),
$$


which is nothing but (2). Different states $\phi, \psi \in \mathcal{D}$ have to share the same $U$ so as to make (12) identically vanish:

$$
\begin{aligned}
\frac{\hbar^{2}}{2 m}\left[\Phi^{\dagger} \Psi^{\prime}-\Phi^{\prime \dagger} \Psi\right] & =\frac{\hbar^{2}}{4 i m L_{0}}\left[\Phi^{(+)^{\dagger}} \Psi^{(+)}-\Phi^{(-)^{\dagger}} \Psi^{(-)}\right] \\
& =\frac{\hbar^{2}}{4 i m L_{0}}\left[\Phi^{(+)^{\dagger}} \Psi^{(+)}-\left(U \Phi^{(+)}\right)^{\dagger}\left(U \Psi^{(+)}\right)\right]=0
\end{aligned}
$$

By an argument analogous to the case of the half line [10], it is not hard to show that the connection condition (2) restricts the space $\mathcal{F}$ to a domain $\mathcal{D} \equiv \mathcal{D}_{U}$ on which the Hamiltonian is not only symmetric but indeed self-adjoint. Since all different $U$ s specify different self-adjoint domains $\mathcal{D}_{U}$, here we can see again that, as in the case of point interactions, the family of self-adjoint Hamiltonians $H \equiv H_{U}$ allowed on the line with potential $V(x)$ possessing a limit-circle singularity and limit-point behavior for $x \rightarrow \pm \infty$ is given by $U(2)$. $U$ will be called the characteristic matrix of the self-adjoint Hamiltonian $H_{U}$.

Various subfamilies of $U(2)$ can be defined analogously to the case of point interactions. For instance, the 'separated subfamily' $\Omega_{3}$ where no probability flow through $x=0$ is allowed is characterized by those $U$ which are diagonal. Indeed, for diagonal $U$ the probability current

$$
j(x)=\frac{\hbar}{2 i m}\left(\psi^{*} \psi^{\prime}-\psi^{\prime *} \psi\right)=\frac{\hbar}{2 i m}\left(W\left[\psi^{*}, \varphi^{(1)}\right] W\left[\psi, \varphi^{(2)}\right]-W\left[\psi^{*}, \varphi^{(2)}\right] W\left[\psi, \varphi^{(1)}\right]\right)
$$

is seen to vanish at $x=0$, and diagonal $U$ s provide the cases when the boundary condition (14) does not mix the +0 boundary values with the -0 ones. Hence we have $\Omega_{3} \simeq$ $U(1) \times U(1) \subset U(2)$, which are, in other words, the cases where the system consists of two independent half line systems.

If the domain of a self-adjoint Hamiltonian contains only functions that are regular at the singularity, then one may choose as reference modes any basis of independent solutions satisfying

$$
\varphi^{(1)}( \pm 0)=0, \quad \varphi^{(1)^{\prime}}( \pm 0)=1, \quad \varphi^{(2)}( \pm 0)=-1, \quad \varphi^{(2)^{\prime}}( \pm 0)=0 .
$$

Under this choice, we find that the boundary vectors (10) reduce to the conventional form (3), which shows that our connection conditions are a natural generalization of the conventional conditions. Once generalized, however, we recognize that the normalizations (17) are not at all essential in presenting the connection conditions (2) at the singularity to ensure the self-adjointness of the Hamiltonian. This in turn suggests that the characteristic matrix $U$ characterizes the singularity only with respect to the reference modes chosen, and this fact has been implicit in the previous treatment for non-singular cases based on the normalizations (17) . 


\section{Two models with singular potential}

We now employ the scheme just presented to analyze the two models mentioned in the Introduction.

\section{(i) One dimensional hydrogen atom}

The first model is the one dimensional hydrogen atom, which is governed by the Coulomb potential,

$$
V(x)=-\frac{e^{2}}{|x|} .
$$

This system has a long history of research, dating back to Loudon [15] who first gave a set of bound state solutions $\left(E_{n}<0\right)$ for the Schrödinger equation,

$$
H \psi_{n}(x)=E_{n} \psi_{n}(x),
$$

in terms of the Whittaker functions. The spectrum obtained in [15] is

$$
E_{n}=-\frac{m e^{4}}{2 \hbar^{2} n^{2}}, \quad n=1,2, \ldots,
$$

where each level is doubly degenerate. The system has later been examined by a number of other groups to obtain different spectra due to different choices of the connection condition at the singularity $x=0$ (see, i.e., $[16,4]$ and references therein). The connection condition adopted originally in [15] is the Dirichlet condition $\psi( \pm 0)=0$ which corresponds to the Friedrichs extension of the Hamiltonian [17], but other extensions are equally possible as we shall now describe.

To apply our scheme of connection conditions, we first recall that in terms of the variables

$$
z=2 \eta x, \quad \eta=\frac{\sqrt{-2 m E_{n}}}{\hbar}, \quad \alpha=\frac{e^{2}}{\hbar} \sqrt{\frac{-m}{2 E_{n}}} .
$$

the Schrödinger equation (19) becomes

$$
\frac{\mathrm{d}^{2} \psi_{n}}{\mathrm{~d} z^{2}}+\left(\frac{\alpha}{|z|}-\frac{1}{4}\right) \psi_{n}=0 .
$$

This is a special case of Whittaker's differential equation, whose two independent solutions are the regular Whittaker function,

$$
M_{\alpha, \frac{1}{2}}(z)=z e^{-\frac{z}{2}} F(1-\alpha, 2 ; z),
$$


where $F(\alpha, \gamma ; z)$ is the confluent hypergeometric function, and the irregular one,

$$
\begin{gathered}
W_{\alpha, \frac{1}{2}}(z)=\frac{e^{-\frac{z}{2}}}{\Gamma(-\alpha)}\{z F(1-\alpha, 2 ; z)[\ln z+\psi(1-\alpha)-\psi(1)-\psi(2)] \\
\left.-\frac{1}{\alpha}+\sum_{r=1}^{\infty} \frac{(1-\alpha)_{r}}{r !(r+1) !} A_{r} z^{r+1}\right\}
\end{gathered}
$$

Here $\Gamma(x)$ is the Gamma function, $\psi(x)=\frac{d}{d x} \ln \Gamma(x)$ is the di-Gamma function, and

$$
A_{r}=\sum_{n=0}^{r-1}\left[\frac{1}{n+1-\alpha}-\frac{1}{n+1}-\frac{1}{n+2}\right], \quad(c)_{r}=\frac{\Gamma(c+r)}{\Gamma(c)}
$$

From the asymptotic behavior of the two solutions, one finds that $W_{\alpha, \frac{1}{2}}(z)$ is square integrable whereas $M_{\alpha, \frac{1}{2}}(z)$ is not. Thus the bound state must be of the form,

$$
\psi_{n}(x)=W_{\alpha, \frac{1}{2}}(|z|)\left\{N_{R} \Theta(x)+N_{L} \Theta(-x)\right\}
$$

where $\Theta(x)$ is the Heaviside step function, and $N_{R}$ and $N_{L}$ are constants to be determined by the connection condition at $x=0$. Note that, since asymptotically

$$
W_{\alpha, \frac{1}{2}}(z)=\frac{1}{\Gamma(-\alpha)}\left\{-\frac{1}{\alpha}+z[\ln z+\psi(1-\alpha)-\psi(1)-\psi(2)]\right\}+\mathcal{O}\left(z^{2} \ln z\right)
$$

as $z \rightarrow 0$, the bound state $\psi_{n}(x)$ has finite limits at $x \rightarrow \pm 0$ whereas the derivative $\psi_{n}^{\prime}(x)$ diverges there.

To see which bound states are actually allowed by the connection condition (2), let us first fix the reference modes $\varphi^{(1)}, \varphi^{(2)}$, in conformity with $(9)$. We choose them as

$$
\begin{aligned}
\varphi^{(1)}(x) & =\frac{1}{2 \kappa} M_{\beta, \frac{1}{2}}(2 \kappa|x|)[\Theta(x)-\Theta(-x)], \\
\varphi^{(2)}(x) & =-\Gamma(1-\beta) W_{\beta, \frac{1}{2}}(2 \kappa|x|),
\end{aligned}
$$

with

$$
\kappa=\frac{\sqrt{-2 m E}}{\hbar}, \quad \beta=\frac{e^{2}}{\hbar} \sqrt{\frac{-m}{2 E}},
$$

which are analogs of (21) with $E_{n}$ replaced by some arbitrary $E<0$. With these, the boundary vectors (10) become finite as they ought to be, and they are proportional to each other,

$$
\Psi=\sigma\left(\begin{array}{c}
N_{R} \\
N_{L}
\end{array}\right), \quad \Psi^{\prime}=\xi \Psi
$$


where

$$
\sigma=\frac{1}{\Gamma(1-\alpha)}, \quad \xi=\frac{2 m e^{2}}{\hbar^{2}}\left[\ln \frac{\alpha}{\beta}-\psi(1-\alpha)+\psi(1-\beta)\right]
$$

With these, the connection conditions (2) read

$$
\left[(U-I)+i L_{0} \xi(U+I)\right] \Psi=0
$$

For $\psi_{n}$ to be a nontrivial bound state, we need

$$
\operatorname{det}\left[U-I+i L_{0} \xi(U+I)\right]=\operatorname{det}\left[D-I+i L_{0} \xi(D+I)\right]=0 \text {, }
$$

where $D$ is a diagonal matrix appearing in the standard decomposition,

$$
U=V^{-1} D V, \quad V \in S U(2) .
$$

In terms of the parameterization,

$$
D=\left(\begin{array}{cc}
e^{i \theta_{+}} & 0 \\
0 & e^{i \theta_{-}}
\end{array}\right), \quad \theta_{ \pm} \in[0,2 \pi)
$$

and

$$
L_{ \pm}=L_{0} \cot \left(\frac{\theta_{ \pm}}{2}\right)
$$

we find that (33) is satisfied if

$$
\xi=-\frac{1}{L_{+}} \quad \text { or } \quad-\frac{1}{L_{-}}
$$

Thus, given the singularity specified by $U$, we can determine the spectrum of the bound states as solutions of (37). We observe that the spectrum depends only on the two angles $\left(\theta_{+}, \theta_{-}\right)$in the diagonal part of $U$, that is, the two eigenvalues of $U$. Later we show that this is in fact the case for all parity invariant potentials $V(-x)=V(x)$ sharing the same singular property considered here.

A particularly simple spectrum is obtained at the angles $\left(\theta_{+}, \theta_{-}\right)=(\pi, \pi)$, i.e., at $U=-I$. The connection condition (32) then implies $\Psi=0$, and therefore we need $\sigma=0$. From (31) we learn that this is fulfilled for $\alpha=1,2, \ldots$, reproducing the spectrum (20) with double degeneracy (since $N_{R}$ and $N_{L}$ are chosen freely modulo the normalization). Note that, since $\varphi^{(1)}( \pm 0)=0$ and $\varphi^{(1)^{\prime}}( \pm 0)=1$, the condition $\Psi=0$ is equivalent to demanding $\psi( \pm 0)=0$, which is obtained by the so called Friedrichs extension discussed in [17]. We, however, emphasize that the Friedrichs extension is a special self-adjoint extension belonging to the separated subfamily $\Omega_{3}$ where the two half lines are physically 
decoupled and none of the scale parameters $L_{ \pm}$in (36) appears in the spectrum, but any other extensions whose spectrum varies with the parameters $\left(\theta_{+}, \theta_{-}\right)$in $U$ through $L_{ \pm}$are equally possible. ${ }^{2}$

We next turn to the scattering phenomena of the Coulomb potential under our general connection conditions. (These considerations will be valid for the repulsive Coulomb force as well, with setting $e^{2}<0$.) For this, in place of $(29)$ we consider a positive scattering energy $E_{k}>0$ and use

$$
k=\frac{\sqrt{2 m E_{k}}}{\hbar}, \quad \gamma=-\frac{e^{2}}{\hbar} \sqrt{\frac{m}{2 E_{k}}} .
$$

Then the two independent solutions are still given by the Whittaker functions with the new $\alpha=i \gamma$ and $z=2 i k|x|$. One may choose the following real combinations for a set of two independent solutions:

$$
\begin{aligned}
\phi_{1}(x) & =\frac{1}{2 i k} M_{i \gamma, \frac{1}{2}}(2 i k|x|)[\Theta(x)-\Theta(-x)], \\
\phi_{2}(x) & =-\frac{1}{2}\left\{\Gamma(1-i \gamma) W_{i \gamma, \frac{1}{2}}(2 i k|x|)+\Gamma(1+i \gamma) W_{-i \gamma, \frac{1}{2}}(-2 i k|x|)\right\} .
\end{aligned}
$$

In passing, we note that the phase part of $\Gamma(1+i \gamma)$, i.e., $\eta_{0}=\arg \Gamma(1+i \gamma)$ is called 's-wave Coulomb phase shift' while its modulus can be evaluated as

$$
|\Gamma(1 \pm i \gamma)|=\sqrt{\Gamma(1+i \gamma) \Gamma(1-i \gamma)}=\sqrt{\frac{2 \pi \gamma}{e^{\pi \gamma}-e^{-\pi \gamma}}}
$$

The set (39) is chosen so that the solutions satisfy $W\left[\phi^{(1)}(x), \phi^{(2)}(x)\right]=1$ and normalized as

$$
\phi_{1}( \pm 0)=0, \quad \phi_{1}^{\prime}( \pm 0)=1, \quad \phi_{2}( \pm 0)=-1,
$$

whereas $\phi_{2}^{\prime}( \pm 0)$ are divergent and cannot be normalized. From (23) and (27) the asymptotic forms of the solutions for $|x| \rightarrow \infty$ are found to be

$$
\begin{aligned}
& \phi_{1}(x) \sim \frac{e^{\frac{\pi}{2} \gamma}}{|\Gamma(1+i \gamma)|} \frac{1}{k} \sin \left(k|x|-\gamma \ln 2 k|x|+\eta_{0}\right)[\Theta(x)-\Theta(-x)], \\
& \phi_{2}(x) \sim-|\Gamma(1+i \gamma)| e^{-\frac{\pi}{2} \gamma} \cos \left(k|x|-\gamma \ln 2 k|x|+\eta_{0}\right) .
\end{aligned}
$$

The general solution for scattering states is a linear combination of the two solutions,

$$
\psi(x)=\left\{N_{R}^{(1)} \phi_{1}(x)+N_{R}^{(2)} \phi_{2}(x)\right\} \Theta(x)+\left\{N_{L}^{(1)} \phi_{1}(x)+N_{L}^{(2)} \phi_{2}(x)\right\} \Theta(-x) .
$$

2 The four-parameter family of extensions for the one dimensional Coulomb system has been argued in [16] in a slightly different scheme. 
Now, from the asymptotic behaviors (42) one deduces that the incoming wave from the left is the one that behaves for $x \pm \infty$ as

$$
\begin{aligned}
\psi(x) & \sim T e^{i(k x-\gamma \log 2 k x)} & & (x \rightarrow+\infty) \\
& \sim e^{i(k x+\gamma \ln (-2 k x))}+R e^{-i(k x+\gamma \ln (-2 k x))} & & (x \rightarrow-\infty) .
\end{aligned}
$$

This corresponds to the choice

$$
N_{R}^{(1)}=i k e^{-\frac{\pi}{2} \gamma-i \eta_{0}}|\Gamma(1+i \gamma)| T, \quad N_{R}^{(2)}=-\frac{e^{\frac{\pi}{2} \gamma-i \eta_{0}}}{|\Gamma(1+i \gamma)|} T
$$

and

$$
N_{L}^{(1)}=-i k e^{-\frac{\pi}{2} \gamma-i \eta_{0}}|\Gamma(1+i \gamma)|\left(R-e^{2 i \eta_{0}}\right), \quad N_{L}^{(2)}=-\frac{e^{\frac{\pi}{2} \gamma-i \eta_{0}}}{|\Gamma(1+i \gamma)|}\left(R+e^{2 i \eta_{0}}\right)
$$

To implement the connection condition at $x=0$, we need to find a set of reference modes, $\varphi^{(1)}(x)$ and $\varphi^{(2)}(x)$ satisfying (9). For this we shall use the same set (42) of the solutions, $\varphi^{(i)}(x)=\phi_{i}(x)$ for $i=1$, 2, with reference energy $E$ and, accordingly, with the corresponding parameter $\gamma(E)$ obtained from (38). The boundary vectors (10) can then be evaluated as

$$
\Psi=-\left(\begin{array}{c}
N_{R}^{(2)} \\
N_{L}^{(2)}
\end{array}\right), \quad \Psi^{\prime}=\left(\begin{array}{c}
N_{R}^{(1)} \\
-N_{L}^{(1)}
\end{array}\right)+\rho\left(\begin{array}{c}
N_{R}^{(2)} \\
N_{L}^{(2)}
\end{array}\right)
$$

with

$$
\rho=\frac{m e^{2}}{\hbar^{2}}\left\{f(E)-f\left(E_{k}\right)\right\}, \quad f(s)=\{2 \ln \gamma(s)-\psi(1-i \gamma(s))-\psi(1+i \gamma(s))\}
$$

Plugging the vectors (47) into the connection condition (2), and solving for the scattering matrix, one obtains

$$
\left(\begin{array}{l}
T \\
R
\end{array}\right)=\frac{-e^{2 i \eta_{0}}}{(U-I)-\omega L_{0}(U+I)}\left[(U-I)+\omega^{*} L_{0}(U+I)\right]\left(\begin{array}{l}
0 \\
1
\end{array}\right)
$$

where we have used

$$
\omega=k e^{-\frac{\pi}{2} \gamma}|\Gamma(1+i \gamma)|^{2}+i \rho e^{\frac{\pi}{2} \gamma} .
$$

Note that the Friedrichs extension $U=-I$ allows no transmission $T=0$. In fact, as seen easily in (49), this is the case for any diagonal $U$, which is expected from the fact that those $U$ belong to the separated subfamily $\Omega_{3}$. 
To render the scattering data (49) more explicit, we use the decomposition (34) with $D$ given in (35) and $V$ parametrized as

$$
V=e^{i \frac{\mu}{2} \sigma_{2}} e^{i \frac{\nu}{2} \sigma_{3}}, \quad \mu \in[0, \pi], \quad \nu \in[0,2 \pi)
$$

Then, in terms of the scale parameters (36) and

$$
\chi_{ \pm}=\arg \left(1+i \omega L_{ \pm}\right)
$$

the scattering formula reads

$$
\left(\begin{array}{c}
T \\
R
\end{array}\right)=-e^{i\left(2 \eta_{0}+\chi_{+}+\chi_{-}\right)}\left(\begin{array}{c}
i \sin \left(\chi_{+}-\chi_{-}\right) \sin \mu e^{-i \nu} \\
\cos \left(\chi_{+}-\chi_{-}\right)-i \sin \left(\chi_{+}-\chi_{-}\right) \cos \mu
\end{array}\right) .
$$

We note that this outcome depends on the choice of the reference modes, not only on the matrix $U$. This is a consequence of the fact that the combination of $U$ and the reference modes, not each, is essential for the determination of the singularity, as mentioned earlier. The ambiguity in the choice of the reference modes is described by the group $S L(2, \mathbb{R})$ on account of the reality condition of the modes and the normalization in the Wronskian (9). Precisely which combinations of the parameters are physically important is an interesting question and will be discussed elsewhere.

\section{(ii) Harmonic oscillator with inverse square potential}

Our second model is the harmonic oscillator with inverse square potential,

$$
V(x)=\frac{m \omega^{2}}{2} x^{2}+g \frac{1}{x^{2}}
$$

In contrast to the previous example, we consider the repulsive case $g>0$ to examine the positively divergent potential, and add the quadratic term to render the entire spectrum discrete. To comply with the condition that the singularity be of the limit-circle case at $x=0$, we confine ourselves to

$$
0<g<\frac{3 \hbar^{2}}{8 m}
$$

for which the Hamiltonian admits a $U(2)$ family of extensions. To solve the Schrödinger equation (19), let us set

$$
\psi_{n}(x)=y^{a+1 / 2} e^{-y^{2} / 2} f_{n}\left(y^{2}\right), \quad y=\sqrt{\frac{m \omega}{\hbar}} x
$$


for $x>0$, and use

$$
a=\frac{1}{2} \sqrt{1+\frac{8 m g}{\hbar^{2}}}, \quad z=y^{2},
$$

so that (19) becomes

$$
z \frac{\mathrm{d}^{2} f_{n}}{\mathrm{~d} z^{2}}(z)+(a+1-z) \frac{\mathrm{d} f_{n}}{\mathrm{~d} z}(z)-\frac{1}{2}\left(a+1-\lambda_{n}\right) f_{n}(z)=0, \quad \lambda_{n}=\frac{E_{n}}{\hbar \omega} .
$$

This is just the confluent hypergeometric differential equation, and hence the two independent solutions for (19) are

$$
\begin{array}{ll}
\phi_{n}^{(1)}(x):=y^{c_{1}-1 / 2} e^{-y^{2} / 2} F\left(\frac{c_{1}-\lambda_{n}}{2}, c_{1} ; y^{2}\right), & c_{1}=1+a, \\
\phi_{n}^{(2)}(x):=y^{c_{2}-1 / 2} e^{-y^{2} / 2} F\left(\frac{c_{2}-\lambda_{n}}{2}, c_{2} ; y^{2}\right), & c_{2}=1-a .
\end{array}
$$

Since the solution for $x<0$ can be found by setting $x \rightarrow-x$ in (59), the general solution for the bound state is given by

$$
\psi_{n}(x)=\left[N_{\mathrm{R}}^{(1)} \phi_{n}^{(1)}(|x|)+N_{\mathrm{R}}^{(2)} \phi_{n}^{(2)}(|x|)\right] \Theta(x)+\left[N_{\mathrm{L}}^{(1)} \phi_{n}^{(1)}(|x|)+N_{\mathrm{L}}^{(2)} \phi_{n}^{(2)}(|x|)\right] \Theta(-x),
$$

where the constants $N_{\mathrm{R}}^{(s)}$ and $N_{\mathrm{L}}^{(s)}$ will be restricted by the connection condition. Note that (55) guarantees that both of the two solutions are square integrable near the singularity. The entire square integrability is then ensured if the solutions vanish sufficiently fast at the infinity $x \rightarrow \pm \infty$. From the asymptotic behavior of the confluent hypergeometric function,

$$
F(\alpha, \gamma ; z) \approx \frac{\Gamma(\gamma)}{\Gamma(\alpha)} e^{z} z^{\alpha-\gamma}, \quad \text { as } \quad|z| \rightarrow \infty
$$

the square integrability of the solutions (59) implies

$$
\frac{N_{\mathrm{R}}^{(1)}}{N_{\mathrm{R}}^{(2)}}=\frac{N_{\mathrm{L}}^{(1)}}{N_{\mathrm{L}}^{(2)}}=-\frac{\Gamma\left(\left(c_{1}-\lambda_{n}\right) / 2\right)}{\Gamma\left(\left(c_{2}-\lambda_{n}\right) / 2\right)} \frac{\Gamma\left(c_{2}\right)}{\Gamma\left(c_{1}\right)} .
$$

Now for the reference modes (9), we choose two eigenmodes belonging to an arbitrarily fixed energy $E$, given in terms of the solutions (59), as

$$
\begin{aligned}
\varphi^{(1)}(x) & =\sqrt{\frac{\hbar}{m \omega}} \phi^{(1)}(|x|)[\Theta(x)-\Theta(-x)], \\
\varphi^{(2)}(x) & =\frac{1}{c_{2}-c_{1}} \phi^{(2)}(|x|) .
\end{aligned}
$$


From $F(\alpha, \gamma ; z)=1+\mathcal{O}(z)$ as $z \rightarrow 0$, the boundary vectors (10) are found to be

$$
\Psi=\left(c_{1}-c_{2}\right)\left(\begin{array}{c}
N_{\mathrm{R}}^{(2)} \\
N_{\mathrm{L}}^{(2)}
\end{array}\right), \quad \Psi^{\prime}=\sqrt{\frac{m \omega}{\hbar}}\left(\begin{array}{c}
N_{\mathrm{R}}^{(1)} \\
N_{\mathrm{L}}^{(1)}
\end{array}\right),
$$

which are finite despite that the solution (60) is divergent at the singularity. Moreover, we observe from the relations (62) and (64) that the two vectors $\Psi^{\prime}$ and $\Psi$ are again proportional to each other, and hence if we write $\Psi^{\prime}=\xi \Psi$ the boundary condition (2) becomes (32) as before. Combining (62) and (64), one finds

$$
\xi=\frac{1}{c_{2}-c_{1}} \sqrt{\frac{m \omega}{\hbar}} \frac{\Gamma\left(\left(c_{1}-\lambda_{n}\right) / 2\right)}{\Gamma\left(\left(c_{2}-\lambda_{n}\right) / 2\right)} \frac{\Gamma\left(c_{2}\right)}{\Gamma\left(c_{1}\right)}
$$

and the spectrum $\left\{E_{n}=\lambda_{n} \hbar \omega\right\}$ is determined by the same condition as in (37). Note that, again, the spectrum depends only on the two angle parameters $\left(\theta_{+}, \theta_{-}\right)$.

At some points of the angles, the spectrum becomes particularly simple. For instance, at $\left(\theta_{+}, \theta_{-}\right)=(0,0)$ (i.e., $\left.U=I\right)$, we obtain $E_{n}=\left(2 n+c_{2}\right) \hbar \omega$ and that the eigenstates are given by $\phi_{n}^{(2)}(|x|)$ both on the positive and negative half lines (and hence each level is doubly degenerate). Similarly, at $\left(\theta_{+}, \theta_{-}\right)=(\pi, \pi)$ (i.e., $\left.U=-I\right)$, we find $E_{n}=\left(2 n+c_{1}\right) \hbar \omega$ and that the eigenstates are $\phi_{n}^{(1)}(|x|)$ which are also doubly degenerate. As mentioned earlier, this corresponds to the Friedrichs extension and has been conventionally considered for the quantization of the system since Calogero [19]. On the other hand, at $\left(\theta_{+}, \theta_{-}\right)=(0, \pi)$, then we have two series of eigenstates, one with $N_{\mathrm{R}}^{(2)}=N_{\mathrm{L}}^{(2)}=0$ and the other with $N_{\mathrm{R}}^{(1)}=N_{\mathrm{L}}^{(1)}=0$, with eigenvalues

$$
E_{n}^{(1)}=(2 n+1+a) \hbar \omega, \quad E_{n}^{(2)}=(2 n+1-a) \hbar \omega, \quad n=0,1, \ldots,
$$

respectively. In particular, in the limit $g \rightarrow 0$ we have $a \rightarrow 1 / 2$, which shows that our system recovers the spectrum of a harmonic oscillator. A complete reduction to the harmonic oscillator system is realized by choosing $U=\sigma_{1}$, where the eigenstates become $e^{-y^{2} / 2}$ times the familiar Hermite polynomials (for a detailed discussion on the smooth limit to the harmonic oscillator, see [3]). In this respect, the extension provided by $U=\sigma_{1}$ causes no obstacle at the singularity and is called 'the free case' in the analysis of point interactions. 


\section{Spectral space for parity invariant singular potentials}

The previous two examples share the property that the spectrum of the Hamiltonian is dependent only on the two parameters $\left(\theta_{+}, \theta_{-}\right)$which are determined by the eigenvalues of the characteristic matrix $U \in U(2)$. This has been observed also for point interaction [18], and can be shown to hold for any singular potential $V(x)$ characterized by $U(2)$, as long as it is parity invariant $V(-x)=V(x)$. Indeed, we have the following

Theorem. If the Schrödinger operator $H$ on $\mathbb{X} \backslash\{0\}$ has a (measurable and locally integrable) parity invariant potential $V(x)=V(-x)$, and is in the limit-circle case at $x=0$ and in the limit-point case for $|x| \rightarrow \infty$, then its spectrum on a self-adjoint domain $\mathcal{D}_{U}$ is uniquely determined by the eigenvalues of the characteristic matrix $U \in U(2)$.

Proof. The proof is done simply by putting the argument of the examples in the general context. Let $\psi_{n} \in L^{2}(\mathbb{X})$ be a normalizable solution of the Schrödinger equation (19) with eigenvalue $E_{n}$, which is subject to the boundary condition (2) specified by the matrix $U$. Let also $\left\{\phi_{n}^{(s)}(x)\right\}_{s=1,2}$ be a fundamental system of real solutions for $x>0$ with the same $E_{n}$. These solutions are not necessarily subject to (2), and are chosen to satisfy $W\left[\phi_{n}^{(1)}, \phi_{n}^{(2)}\right]=1$. In terms of these, the general solution of $\psi_{n}$ can be given in the form (60) because of the parity invariance of the potential, $V(-x)=V(x)$. Since (at least one but generically both of) the basis solutions $\phi_{n}^{(s)}(x)$ become divergent as $x \rightarrow \infty$ as dictated by the uniqueness of the solution at the limit-point infinity, one needs to arrange the coefficients, $N_{\mathrm{R}}^{(s)}$ and $N_{\mathrm{L}}^{(s)}$, so that the divergence of the two terms cancel each other in the limits $x \rightarrow \pm \infty$. From this one deduces the equality of the two ratios $N_{\mathrm{R}}^{(1)}: N_{\mathrm{R}}^{(2)}=N_{\mathrm{L}}^{(1)}: N_{\mathrm{L}}^{(2)}$, that is,

$$
N_{\mathrm{R}}^{(2)}=\alpha N_{\mathrm{R}}^{(1)}, \quad N_{\mathrm{L}}^{(2)}=\alpha N_{\mathrm{L}}^{(1)}
$$

with some $\alpha \in \mathbb{R} \cup\{\infty\}$. We also define our reference modes (9) as

$$
\begin{aligned}
& \varphi^{(1)}(x)=\phi^{(1)}(|x|)[\Theta(x)-\Theta(-x)], \\
& \varphi^{(2)}(x)=\phi^{(2)}(|x|),
\end{aligned}
$$

using two real eigenmodes $\phi^{(1)}, \phi^{(2)}$ for $x>0$ corresponding to an arbitrary eigenvalue $E$, satisfying $W\left[\phi^{(1)}, \phi^{(2)}\right]=1$. Then, one obtains the following relations for the Wronskians:

$$
W\left[\phi_{n}^{(s)}, \varphi^{(1)}\right]_{+0}=W\left[\phi_{n}^{(s)}, \varphi^{(1)}\right]_{-0}, \quad W\left[\phi_{n}^{(s)}, \varphi^{(2)}\right]_{+0}=-W\left[\phi_{n}^{(s)}, \varphi^{(2)}\right]_{-0} .
$$


With the help of these relations (69) and (67), one can compute the boundary vectors (10) to find

$$
\begin{gathered}
\Psi=\left(W\left[\phi_{n}^{(1)}, \varphi^{(1)}\right]_{+0}+\alpha W\left[\phi_{n}^{(2)}, \varphi^{(1)}\right]_{+0}\right)\left(\begin{array}{c}
N_{\mathrm{R}}^{(1)} \\
N_{\mathrm{L}}^{(1)}
\end{array}\right), \\
\Psi^{\prime}=\left(W\left[\phi_{n}^{(1)}, \varphi^{(2)}\right]_{+0}+\alpha W\left[\phi_{n}^{(2)}, \varphi^{(2)}\right]_{+0}\right)\left(\begin{array}{c}
N_{\mathrm{R}}^{(1)} \\
N_{\mathrm{L}}^{(1)}
\end{array}\right) .
\end{gathered}
$$

One thus sees that the two complex boundary vectors are proportional to each other, $\Psi^{\prime}=\xi \Psi$, with a constant $\xi$ that is specified by (67) and (70). Once this is established, the rest of the argument is already given in the examples. Namely, the boundary condition (2) now becomes (32) and, hence, a nontrivial solution is obtained if (33) is fulfilled. In terms of the parameters (36) the spectrum condition reads (37). This proves the statement of the theorem for the bound states, since the diagonal slots of $D$ are nothing but the eigenvalues of the matrix $U$. In the end, we recall the fact that the continuous spectrum is independent of $U$, since all self-adjoint extensions of a symmetric operator admit the same continuous spectrum [7]. Q.E.D.

We note that the space of possible spectra is therefore the space of the eigenvalues of $U$, which is $U(1) \times U(1) / \mathbb{Z}_{2}$ (where $\mathbb{Z}_{2}$ is the factor of interchanging the two eigenvalues) forming a Möbius strip with boundary [18]. This theorem implies that, since the separated subfamily $\Omega_{3}$ contains all possible diagonal $U$, the probability flow through the singularity plays no role as long as the spectra of parity invariant systems are concerned. In other words, the variety of the spectra is exhausted by systems consisting of two separated half lines, when all possible conditions at the boundary, i.e., the $U(1)$ family of boundary conditions each, are allowed on both sides. 


\section{Summary and Discussions}

In this paper we presented the generalized connection conditions for singular potentials characterized by the matrix $U \in U(2)$ in the form (2) with improved boundary vectors (10). An essential point in our generalization is the use of Wronskians in the boundary vectors (10) which are well-defined even in the limits to the singularity $x=0$, in contrast to the earlier ones (3) which become ill-defined in the limits. Using the generalized connection conditions, we examined two models, the one dimensional hydrogen atom and the harmonic oscillator with inverse square potential, which are solvable and yet so far have yielded conflicting results in the spectrum. Our analysis shows that the spectrum varies according to the connection conditions adopted, and that the possible spectra form a 2parameter subspace (Möbius strip with boundary) in the entire $U(2)$ family. The confusion on the spectrum is therefore resolved once we understand which connection conditions if formulated in the form (2) - one is using in the analysis. We also note that in our connection conditions the parameters in the matrix $U$, when combined with the decomposition (34), bear direct physical meanings [18]. Indeed, we have already seen this in the spectral theorem in sect. 4 in that the two parameters in the diagonal piece $D$ represent the two independent scales of the system.

As a final remark, we wish to mention that the whole prescription for the connection conditions remains valid even for systems with a 'black box', not just a singular point. Namely, if there is a blank interval $\mathbb{I}=\left[-\varepsilon_{0}, \varepsilon_{0}\right]$ with some small $\varepsilon_{0}>0$ on a line, then its quantum mechanical description can be given by means of our prescription if one replaces $\mathbb{X} \backslash\{0\}$ with $\mathbb{X} \backslash \mathbb{I}$, and the relevant Wronskians to be used in the boundary vectors are $W\left[\phi^{*}, \psi\right]_{ \pm \varepsilon_{0}}$ instead of $W\left[\phi^{*}, \psi\right]_{ \pm 0}$. This seemingly innocent modification has a practical consequence, since this allows us to introduce a rich $U(2)$ structure to strong singularities that are in the limit-point case and are therefore originally essentially self-adjoint with no ambiguity in connection conditions. The appearance of the four-parameter $U(2)$ freedom in choosing a singular potential may provide a useful theoretical framework for describing such singularities in quantum phenomena that seem to belong to the limit-point case by their behavior at intermediate length scales but have a richer structure at very short length scales. We mention that several recent approaches address the question of how to introduce nontrivial structure to limit-point singularities, see, e.g., [20].

Acknowledgement: I.T. is indebted to H. Miyazaki for useful comments. T.C. thanks members of the Theory Group of KEK for the hospitality extended to him during his stay. This work has been supported in part by the Grant-in-Aid for Scientific Research (C) (Nos. 10640301 and 13640413) and that on Priority Areas (No. 13135206) by the Japanese Ministry of Education, Science, Sports and Culture. 


\section{References}

[1] A. Fuhrer, S. Lüsher, T. Ihn, T. Heinzel, K. Ensslin, W. Wegscheider and M. Bichler, Nature 413 (2001) 822.

[2] T. Cheon, T. Fülöp and I. Tsutsui, Ann. Phys. 294 (2001) 1.

[3] H. Miyazaki and I. Tsutsui, Ann. Phys. 299 (2002) 78.

[4] A.N. Gordeyev and S.C. Chhajlany, J. Phys. A 30 (1997) 6893.

[5] L. Lathouwers, J. Math. Phys. 16 (1975) 1393.

[6] M. Reed and B. Simon, "Methods of Modern Mathematical Physics II, Fourier analysis, self-adjointness", Academic Press, New York, 1975.

[7] N.I. Akhiezer and I.M. Glazman, "Theory of Linear Operators in Hilbert Space", Vol.II, Pitman Advanced Publishing Program, Boston, 1981.

[8] G. Bonneau, J. Faraut and G. Valent, Self-adjoint extensions of operators and the teaching of quantum mechanics, LPTHE preprint PAR/LPTHE/99-43, quant$\mathrm{ph} / 0103153$.

[9] T. Fülöp and I. Tsutsui, Phys. Lett. A264 (2000) 366.

[10] A.M. Krall, J. Differential Equations 45 (1982) 128.

[11] S. Albeverio, F. Gesztesy, R. Høegh-Krohn and H. Holden, "Solvable Models in Quantum Mechanics", Springer, New York, 1988.

[12] F. Rellich, Math. Ann. 122 (1951) 343.

[13] C.T. Fulton, Trans. Amer. Math. Soc. 229 (1977) 51; Proc. Roy. Soc. Eddingburgh Sect $A 87$ (1980) 1 .

[14] A.N. Kochubei, Siberian Math. J. 32 (1991) 401.

[15] R. Loudon, Amer. J. Phys. 27 (1959) 649.

[16] W. Fisher, H. Leschke and P. Müller, J. Math. Phys. 36 (1995) 2313.

[17] F. Gesztesy, J. Phys. A 13 (1980) 867.

[18] I. Tsutsui, T. Fülöp and T. Cheon, J. Math. Phys. 42 (2001) 5687.

[19] F. Calogero, J. Math. Phys. 10 (1969) 2191, 2197; 12 (1971) 419.

[20] P. Kurasov and K. Watanabe, On Rank One $\mathrm{H}_{-3}$-Perturbations of Positive Selfadjoint Operators, Stockholm Univ. Res. Rep. Math. 1999/10; On $H_{-4}$-perturbations of self-adjoint operators, Stockholm Univ. Res. Rep. Math. 2000/16. 